\title{
A second generation HIV-IN-EGFP fluorescent viral system to analyze HIV-1 in the nuclear compartment of infected cells
}

\author{
Ashwanth Christopher Francis ${ }^{1,2^{*}}$, Cristina Di Primio ${ }^{3}$, Valentina Quercioli $^{3}$, Annegret Boll ${ }^{1}$, Daniele Arosio ${ }^{2}$, \\ Anna Cereseto ${ }^{1}$ \\ From Frontiers of Retrovirology: Complex retroviruses, retroelements and their hosts \\ Cambridge, UK. 16-18 September 2013
}

\section{Background}

To study the nuclear biology of HIV-1 we recently developed a microscopy based fluorescent HIV-INEGFP system [1]. HIV-IN-EGFP exploits the Vpr mediated trans-incorporation to incorporate IN-EGFP in viral particles made with pNL-IN-D64E. The ability to visualize HIV-IN-EGFP within the nucleus makes it an attractive tool to study quantitatively the nuclear import step of HIV-1 [2,3]. In this work we provide new evidence for efficient visualization of HIV-1 complexes in the nuclei of infected cells through a new optimized system.

\section{Methods}

In order to optimize HIV-IN-EGFP infectivity various modifications were introduced into the visualization system. The functional tetramerization of IN mutations E11K and K186E [4] was introduced into Vpr-IN-EGFP constructs and nuclear detection of functional tetramers of HIV-IN-EGFP was evaluated in Hela-P4 cells. In addition the EGFP was replaced in Vpr-IN with superfolder GFR. Infectivity of HIV-IN-EGFP was verified in $293 \mathrm{~T}$ cells at 3 days post infection.

\section{Results}

Since the HIV-IN-EGFP visualization system [1] is produced by IN-EGFP trans-incorporation through Vpr, we have better analyzed the impact of this manipulation on viral infectivity. We observed that Vpr-INEGFP molecules are able to efficiently complement D64E mutant integrase for infectivity, however the

${ }^{1}$ Centre for Integrative Biology (CIBIO), University of Trento, Trento, Italy Full list of author information is available at the end of the article infectivity values of trans-incorporated viruses are low with respect to the NL4.3 virus. Through trans-complementation of NL4.3 virus we find that incorporation of IN alone or fused with EGFP has a lethal effect in native wild type virions by severely affecting their infectivity. Further, by modifying the tetramerization properties of IN-EGFP [4] we attempted to improve viral infectivity. In fact, as previously suggested by Hare et al. [4], trans-incorporation of integrase molecules mutated in the $\mathrm{N}$-terminus $(\mathrm{E} 11 \mathrm{~K})$ and catalytic domain (K186E) induces stabilization of the integrase tetramer leading to a more functional complex. Confocal microscopic analysis revealed that functional tetramerization of IN-EGFP results in a loss of GFP fluorescence that can be recovered through the use of brighter fluorophores such as the super-folder GFP. Finally, the improved infectivity of the trans-incorporated viral particles was complemented with a dual labeling, which allows monitoring the different monomers within the integrase complex.

\section{Conclusions}

Until recently the nuclear biology of HIV-1 has been investigated through molecular techniques, with the development of HIV-IN-EGFP it is possible to assess the localization of HIV-1 in the nuclear compartment. This technique allowed the first determination that HIV-1 localizes in the de-condensed regions of the chromatin and near the nuclear rim [1]. In this work we exploited improved tetramerization properties of integrase [4] to develop a second-generation HIV-IN-EGFP, which preserves the same imaging properties as the first-generation even though the infectivity is significantly improved. 


\section{Authors' details}

${ }^{1}$ Centre for Integrative Biology (CIBIO), University of Trento, Trento, Italy.

${ }^{2}$ Istituto di Biofisica, Consiglio Nazionale delle Ricerche, Trento, Italy.

${ }^{3}$ Laboratory of Neurobiology, Scuola Normale Superiore, Pisa, Italy.

Published: 19 September 2013

\section{References}

1. Albanese, et al: 2008 .

2. Christ, et al: 2008.

3. Desimmie, et al: 2013.

4. Hare, et al: 2009

doi:10.1186/1742-4690-10-S1-P31

Cite this article as: Francis et al:: A second generation HIV-IN-EGFP

fluorescent viral system to analyze HIV-1 in the nuclear compartment of infected cells. Retrovirology 2013 10(Suppl 1):P31.

Submit your next manuscript to BioMed Central and take full advantage of:

- Convenient online submission

- Thorough peer review

- No space constraints or color figure charges

- Immediate publication on acceptance

- Inclusion in PubMed, CAS, Scopus and Google Scholar

- Research which is freely available for redistribution

Submit your manuscript at www.biomedcentral.com/submit 